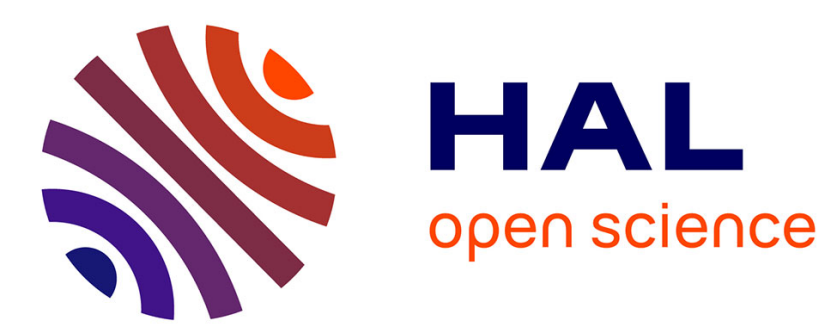

\title{
Le rôle des libelli dans la pratique liturgique du haut Moyen Âge. Histoire et typologie
}

Eric Palazzo

\section{To cite this version:}

Eric Palazzo. Le rôle des libelli dans la pratique liturgique du haut Moyen Âge. Histoire et typologie. Revue Mabillon, revue internationale d'histoire et de littérature religieuses, 1990, 1 (62), pp.9-36. 10.1484/J.RM.2.305467 . halshs-01355069

\section{HAL Id: halshs-01355069 \\ https://shs.hal.science/halshs-01355069}

Submitted on 22 Aug 2016

HAL is a multi-disciplinary open access archive for the deposit and dissemination of scientific research documents, whether they are published or not. The documents may come from teaching and research institutions in France or abroad, or from public or private research centers.
L'archive ouverte pluridisciplinaire HAL, est destinée au dépôt et à la diffusion de documents scientifiques de niveau recherche, publiés ou non, émanant des établissements d'enseignement et de recherche français ou étrangers, des laboratoires publics ou privés. 


\title{
LE ROLE DES LIBELLI DANS LA PRATIQUE LITURGIQUE DU HAUT MOYEN AGE HISTOIRE ET TYPOLOGIE*
}

\author{
$\stackrel{\text { par }}{\text { Éric PALAZZO }}$
}

«Memorandus Heroldus, huius loci frater et monachus, vitas et passiones multorum sanctorum in diversis libellis magno labore congessit. Sed quia parvitas librorum videbatur minus apta cernentibus, et facile poterat furto vel qualibet surrepcione perire res modica, ego auxilio et industria domini Ellenhardi prioris venerandi omnes illos libellos adiunctis quoque aliis vitis et passionibus in quatuor magna volumina redegi $\gg$.

A travers ce texte introductif à l'un des catalogues de l'abbaye de Michelsberg à Bamberg (première moitié du XII' siècle), le moine Burchard a laissé un témoignage, fort personnalisé, sur un aspect de la pratique des bibliothécaires du Moyen Age. Burchard explique très clairement quelles étaient les habitudes, suivies par lui-même, des moines chargés de tenir en ordre la bibliothèque du monastère : les libelli de vies de saints étaient regroupés pour former plusieurs gros volumes. Pendant une grande partie du Moyen Age, plus particulièrement à l'époque carolingienne, les libelli hagiographiques ont été principalement utilisés pour la diffusion de la vie et donc du culte d'un saint ${ }^{2}$.

A côté de ces libelli hagiographiques, le Moyen Age a connu une grande variété de libelli liturgiques dont l'histoire et la typologie n'ont été abordées jusqu'à présent que de façon ponctuelle et partielle. En guise d'introduction à l'exposé de nos recherches, une rapide synthèse des principaux acquis de la science liturgique en

* Je remercie le père Gy et Dominique Iogna-Prat d'avoir relu et enrichi de leurs remarques cet article.

1. K. DENGLER-SCHREIBER, Scriptorium und Bibliothek des Klosters Michelsberg in Bamberg, Graz, 1979, p. 184.

2. Voir notamment B. BISCHOFF, «Über gefaltete Handschriften, vormehmlich hagiographischen Inhalts ", dans Mittelalterliche Studien, Stuttgart, 1966, t. I, p. 93-100 ; G. PHILIPPART, Les légendiers latins et autres manuscrits hagiographiques, Tumhout, 1977 (Typologie des sources du Moyen Age occidental, fasc. 24-25), p. $21 s q ., 99-101$. Bon nombre de ces libelli hagiographiques étaient décorés d'un cycle iconographique se rapportant aux événements majeurs de la vie du saint ou de la sainte ; voir à ce propos l'enquête menée par $F$. WoRMALD pour des libelli réalisés entre le $\mathrm{X}^{\mathrm{e}}$ et la fin du X $\mathrm{XI}^{\mathrm{e}}$ siècle, "Some Illustrated Manuscripts of the Lives of the Saints », dans Bulletin of the John Rylands Library, t. 35, 1952, p. $148-166$.

Revue Mabillon, n.s., t. 1 (= t. 62), 1990, p. 9-36. 
matière de libelli sera proposée. Ce parcours historiographique permettra en même temps de mieux saisir la place des libelli dans l'histoire des livres liturgiques et de leur typologie.

Les enquêtes menées à partir de différentes sources textuelles (notamment des textes liturgiques, mais surtout les catalogues médiévaux de bibliothèques) ont permis d'approfondir et d'affiner notre perception des désignations médiévales de ces libelli. Les résultats lexicographiques obtenus augmentent notablement la connaissance de ces documents, surtout par une meilleure compréhension de leur aspect codicologique ainsi que de leur contenu ${ }^{3}$.

Après avoir exploré les sources textuelles, il faudra nous tourner vers les libelli eux-mêmes afin d'examiner, tout d'abord, la correspondance entre les désignations relevées dans les textes et les manuscrits parvenus jusqu'à nous. Les nombreux cas présentés permettront ensuite d'établir une typologie du libellus liturgique.

A partir de cette typologie, nous essayerons d'éclairer le rôle de ces documents dans la pratique liturgique du haut Moyen Age, notamment dans les monastères, pour le service épiscopal et, de façon plus incertaine, pour le prêtre de paroisse. Nous verrons aussi de quelle façon les libelli ont influencé, et même développé, certaines pratiques liturgiques. Dans quel cadre et pour quelle action liturgique les utilisait-on, et avec quelle fréquence ? Autant de questions auxquelles nous tenterons de répondre afin de restituer le cadre vivant de la liturgie médiévale 4 .

3. Depuis longtemps, les liturgistes ont souligné l'importance, pour la typologie des livres liturgiques, d'une désignation, tant médiévale que moderne, aussi précise que possible de tout manuscrit. Malheureusement, ce souci ne fut que relativement peu suivi par les chercheurs. Parmi les meilleurs exemples en matière de désignation typologique, on compte, bien sûr, les catalogues du chanoine Leroquais dont on pourra lire aussi la leçon d'ouverture à l'École Pratique des Hautes Études, tenue le 13 novembre 1931 (13 p.), les nombreux travaux de Klaus Gamber sur les sacramentaires, souvent à juste titre controversés, ainsi que diverses publications du père Gy (« Typologie et ecclésiologie des livres liturgiques », dans La Maison-Dieu, $\mathrm{n}^{\circ} 121,1975$, p. 7-21, rééd. dans La liturgie dans l'histoire, Paris, 1990, p. 75-89) dont la thèse sur les « Rituels manuscrits des bibliothèques publiques de France » est restée inédite. En ce qui concerne les noms latins donnés aux différents livres liturgiques, le père Gy a souligné qu'il s'agissait d'une question mal étudiée, cf. son article : « Le vocabulaire liturgique latin au Moyen Age », dans La lexicographie du latin médiéval et ses rapports avec les recherches actuelles sur la civilisation du Moyen Age, Paris, 1981, p. 295-301, spéc. 300-301. Notre modeste enquête sur les termes utilisés dans les catalogues médiévaux pour désigner les libelli nous a fait entrevoir l'intérêt d'une étude plus vaste concernant l'ensemble des livres liturgiques.

4. Grâce à la confrontation de différentes sources (textes littéraires, textes liturgiques, témoignages archéologiques), Victor Saxer a récemment mené une étude très stimulante sur l'histoire des usages et des modes liturgiques à Rome dans l'Antiquité et le haut Moyen Age, restituant ainsi les aspects vivants du culte chrétien, cf. V. SAXER, «L'utilisation par la liturgie de l'espace urbain et suburbain : l'exemple de Rome dans l'Antiquité et le haut Moyen Age ", dans Actes du 


\section{Les libelli dans l'histoire de la liturgie}

La distinction entre libelli et grands volumes est ancienne puisque, déjà au XVIII' siècle, le liturgiste italien F.A. Zaccaria établissait des catégories de livres liturgiques ${ }^{5}$ où étaient séparées les petites et les grandes entités livresques ${ }^{6}$. Voici la définition du libellus donnée par Zaccaria : "Praeter missales libros, quos hactenus numeravimus, aliqui sunt qui paucas tantum missas continetur ». A l'intérieur de la même catégorie de petits volumes, Zaccaria définissait les quaterniones : "Idem Tarvisinae Ecclesiae indices nobis exhibent quaternum pro mortuis, quaternionem scilicet, aut libellum, in quo missae pro defunctis. "

Les recherches modernes sur les libelli liturgiques ont été marquées par les travaux de A. Stuiber, du père Gy, de M. Huglo et, plus récemment, de N.K. Rasmussen, à côté desquels prennent place plusieurs études de cas vérifiant et illustrant, pour la plupart, les grandes idées émises par ces chercheurs. Tout d'abord, le libellus est fondamental pour comprendre la formation de certains livres liturgiques. A. Stuiber a montré que le sacramentaire Léonien résultait d'un travail de compilation de libelli réalisé dans le courant du VI ${ }^{e}$ siècle ${ }^{7}$. De son côté, N.K. Rasmussen a éclairé la genèse du livre liturgique de l'évêque, le pontifical, à partir de la réunion puis de la fusion, plus ou moins réfléchies, d'ordines transcrits dans différents libelli ${ }^{8}$. Dans le domaine des livres de chant, M. Huglo a à maintes reprises établi le rôle des libelli dans la formation de certains d'entre eux. Par exemple, dans la seconde moitié du IXe siècle, à Saint-Gall, Notker le Bègue transcrivit ses versets d'hymnes et de séquences dans un libellus qu'il désigna comme tel

$X I^{e}$ Congrès d'Archéologie chrétienne, Lyon-Vienne-Genève-Grenoble et Aoste (2128 septembre 1986), Rome, 1989, p. 917-1031. Après avoir édité les textes majeurs de l'histoire de la liturgie, recherché leur archétype, écrit leur histoire, il faut souhaiter que les liturgistes s'orientent maintenant vers ce type d'étude, d'un grand intérêt pour l'historien.

5. Sur l'histoire même du mot libellus et ses multiples acceptions, notamment dans le domaine du droit romain, cf. l'article du Thesaurus linguae latinae, VI/2, col. 1262-1270. On abordera un peu plus loin le sens « bibliographique » du mot qui a certaines incidences sur notre recherche.

6. F.A. ZACCARIA, Bibliotheca Ritualis, Roma, 1776, vol. I, p. 75-76.

7. Le témoin le plus ancien du Léonien est conservé à Vérone (Biblioteca Capitolare, cod. LXXXVI) et date du VII ${ }^{e}$ siècle ; A. STUIBER, Libelli Sacramentorum Romani, Bonn, 1950, spéc. p. 79-82 ; voir aussi J. DESHUSSES, « Les sacramentaires. Etat actuel de la recherche », dans Archiv für Liturgiewissenschaft, Jhrg. 24, 1982, p. 19-46, spéc. $24-26$.

8. N.K. RASMUSSEN, Les pontificaux du haut Moyen Age, Doctorat en théologie de I'Institut catholique de Paris, 3 vol., Paris, 1978 (édition en préparation par le P. Gy et l'auteur de cet article dans la collection du Spicilegium Sacrum Lovaniense); sur la typologie du libellus liturgique, cf. t. III, p. 431-436. Dans l'exposé des différents cas de libelli, nous reviendrons sur certains exemples traités par Rasmussen. 
dans la lettre-préface à son liber hymnorum envoyé, en 884 , à Liutward de Verceil 9 . Un peu à l'image des pontificaux, les premiers tropaires-prosaires résultent, eux aussi, de la compilation de riches séries de tropes, auparavant diffusées par l'intermédiaire de libelli ${ }^{10}$.

Les différentes recherches évoquées ont par ailleurs mis en évidence le but très souvent pratique du libellus liturgique. En effet, l'usage de créer des textes liturgiques pour diverses circonstances (ordo, office, tropes, formulaires de messes...) favorisa la confection de libelli destinés à la diffusion du nouveau texte. Les messes votives composées par Alcuin, dont nous reparlerons plus loin, illustrent à merveille ce cas de figure.

Pour notre sujet, l'apport du P. Gy est double, à la fois «pratique » et « théorique ». Sa thèse sur les rituels manuscrits, dont il a donné un condensé dans un important article ${ }^{11}$, a fait découvrir une série de libelli destinés à telle ou telle action liturgique, puisqu'ils contiennent tout ce qui est nécessaire au déroulement d'un rite particulier ${ }^{12}$. A la différence des libelli de tropes ou de formulaires de messes, ceux-ci n'ont jamais été réunis dans le but de former un livre liturgique à part entière. La raison en est simple : leur contenu les faisait utiliser indépendamment les uns des autres, chacun pour le rituel pour lequel il avait été conçu. Nous reviendrons sur ce point très important qui touche à la question du rôle des libelli dans la pratique liturgique. Le P. Gy a par ailleurs proposé un certain nombre de réflexions générales sur les libelli ${ }^{13}: 1$. le libellus comporte un seul cahier ou tout au plus deux à trois quaternions; 2 . à l'origine, ces cahiers étaient indépendants ; 3 . le libellus n'est pas relié ; 4. au point de vue liturgique, le libellus a pour objet non pas l'ensemble des fonctions d'un ministre, ou l'ensemble de l'année liturgique, mais seulement une fête déterminée ou une action liturgique (agenda) particulière.

9. Iste libellus habet versus modulaminis apti, cf. M. HUGLo, "Codicologie et musicologie », dans Miscellanea codicologica F. Masai dicata, t. I, Gand, 1979, p. 71-82, spéc. $72-73$.

10. M. Huglo, «Les libelli de tropes et les premiers tropaires-prosaires », dans Pax et Sapientia, Studies in Text and Music of Liturgical Tropes and Sequences in Memory of Gordon Anderson, Stockholm, 1986, p. 13-22, et ID., Les livres de chant liturgique, Turnhout, 1988 (Typologie des sources du Moyen Age occidental, fasc. 52), p. 64-75.

11. P.-M. GY, "Collectaire, rituel, processionnal », dans Revue des Sciences philosophiques et théologiques, t. 44, 1960, p. 441-469, repris dans La liturgie dans l'histoire, op. cit. en note 3, p. 91-126.

12. P.-M. GY, art. cit. note précédente, p. 457-459.

13. Lettre du 29 février 1980 adressée à N.K. Rasmussen, cf. P.-M. GY, « Les différentes formes de libelli liturgiques ", dans Mélanges en l'honneur de N.K. Rasmussen, sous presse. 
A côté de considérations d'ordre codicologique, le quatrième point du P. Gy nous semble fondamental pour comprendre le rôle de ce type de sources dans la pratique liturgique. L'examen de nombreux manuscrits montrera l'intérêt de cet aspect de la définition proposée.

Avant de clore cette brève introduction à l'historiographie du libellus liturgique, ajoutons que la période concernée par l'enquête s'étend des $\mathrm{V}^{\mathrm{e}}-\mathrm{VI}^{\mathrm{e}}$ aux $\mathrm{X}^{\mathrm{e}}$-XI⿳亠口冋丁口 siècles, avec quelques incursions aux $\mathrm{XI}^{e}$ et XII'e siècles. C'est en effet au cours de ce long moment de l'histoire de l'Occident que les livres liturgiques de «première génération » (sacramentaires, antiphonaires, pontificaux, rituels...) prennent forme avec, parmi eux, les libelli, avant d'être supplantés à partir du XIe, mais surtout du XII ${ }^{e}$ siècle, par le missel, ainsi que par l'apparition d'autres livres plus adaptés aux nouvelles pratiques liturgiques, particulièrement à la Curie romaine ${ }^{14}$.

\section{Le témoignage des textes littéraires et liturgiques}

Un célèbre passage de l'Historia Francorum de Grégoire de Tours constitue, à notre connaissance, le plus ancien témoignage littéraire de l'emploi du terme libellus pour désigner un livre servant au culte. A propos de saint Sidoine, évêque de Clermont au milieu du ve siècle, Grégoire écrit :

"Contigit autem quadam die ut ad festivitatem basilicae monasterii invitatus accederet, ablatoque sibi nequiter libello per quem sacrosancta sollemnia agere consuererat, ita paratus a tempore cunctum festivitatis opus explicuit, ut ab omnibus miraretur $\$^{15}$.

Étant donné l'époque ancienne à laquelle se situe cet épisode, on peut penser que l'évêque de Clermont utilisait bel et bien un libellus, car les livres liturgiques complets ne firent leur apparition, de façon certaine, qu'à partir du VIIe siècle. Les témoignages à peu près contemporains de Gennade de Marseille sur les livres dont il attribue la composition à saint Paulin de Nole, à l'évêque Voconius (mort en 460) et au prêtre Musée (mort vers 460), bien que moins clairs que celui de Grégoire de Tours, n'excluent pas la désignation de possibles libelli ${ }^{16}:$ "Pastor episcopus composuit libellum in modum symboli parvum, totam pene ecclesiasticam credulitatem per sententias continentem $\gg^{17} ;$ «... sed et ad personam sancti

14. Cf. P.-M. GY, « Typologie et ecclésiologie des livres liturgiques », art. cit. en note 3 .

15. Historia Francorum, II, 22.

16. C'est aussi l'opinion récente de J. Deshusses, art. cit. en note 7, p. 22.

17. P.L., 58, col. 1103 . 
Eustachii episcopi, successoris supradicti hominis Dei, composuit sacramentorum egregium non parvum volumen, per membra quidem pro opportunitate officiorum et temporum, pro lectionum textu... » ${ }^{18}$; pour Paulin de Nole : "fecit sacramentorum et hymnarium $\gg 19$.

Avant d'examiner les témoignages de l'époque carolingienne, arrêtons-nous un instant sur les difficultés d'interprétation du terme même, soulevées par les exemples précédents. Dans un récent article consacré aux noms de livres, François Dolbeau a attiré l'attention sur les problèmes posés par les interférences entre le domaine intellectuel et celui de la codicologie ${ }^{20}$. En effet, le livre est à la fois une unité " catalographique », intellectuelle et bibliographique, si bien que les hommes du Moyen Age le nomment souvent par son contenu. De toute évidence, ces difficultés existent pour la désignation des livres liturgiques, et plus particulièrement pour les libelli; nous y reviendrons à propos des témoignages des catalogues médiévaux.

Dans une de ses lettres, Alcuin établit la distinction entre les libelli et les sacramentaires de grandes dimensions : "De ordinatione et dispositione missalis libelli nescio cur demandasti. Numquid non habes Romano more ordinatos libellos sacratorios abundanter? Habes quoque et veteris consuetudinis sufficienter sacramentaria maiora ${ }^{21}$. Dans deux autres de ses lettres, le liturgiste carolingien atteste qu'il fait lui-même usage de livrets (certainement des libelli) pour diffuser les messes qu'il a composées $^{22}$. Voici, par exemple, le début de celle envoyée aux moines de Fulda : "Misi cartulam missalem vobis, o sanctissimi presbiteri... $\$^{23}$. Lorsque l'on sait la place prééminente occupée par Alcuin dans le monde carolingien, en particulier dans le domaine de la liturgie, ces deux témoignages, malgré leur isolement, n'en acquièrent pas moins une importance considérable.

D'autant plus que, si l'on regarde maintenant du côté des textes liturgiques à proprement parler, on reste presque autant sur sa faim. Seul un ordo du Pontifical romano-germanique, vraisemblablement antérieur à la composition de ce dernier dans la seconde moitié du

18. Ibid., col. 1104.

19. Ibid., col. 1087.

20. F. DOLBEAU, « Noms de livres ", dans Vocabulaire du livre et de l'écriture au Moyen Age, Crvicima, Turnhout, 1989 (Études sur le vocabulaire intellectuel au Moyen Age, 2), p. 79-99; sur l'emploi de libellus, p. 88-89 et 93-94.

21. M.G.H., Epistolae, IV, Aevi Karolini, II, 1895, p. 370.

22. Sur ces messes, cf. J. DESHuSsES, « Les messes d'Alcuin ». dans Archiv für Liturgiewissenschaft, Bd 14, 1972, p. 7-41.

23. M.G.H., op. cit. en note 21 , p. 404-406. 
$\mathrm{X}^{\mathrm{e}}$ siècle ${ }^{24}$, puis repris, entre autres, dans le Pontifical romain du $\mathrm{XII}^{e}$ siècle, commence par le texte suivant : "Ordinatio exorcistarum. Exorcistae cum ordinantur accipiant de manu episcopi libellum in quo scripti sunt exorcismi ... »25. Le libellus en question apparaît ici comme le futur instrument de travail de l'exorciste.

Cette absence presque totale du mot libellus ou d'un terme assimilé n'a pas lieu de surprendre. D'une manière générale, les textes liturgiques, principalement les ordines, par définition très descriptifs des rites, ne nomment que très rarement les livres utilisés dans l'exercice même du culte. Les désignations demeurent d'ailleurs le plus souvent assez floues, et trop imprécises pour l'historien de la liturgie. Les nombreuses mentions contenues dans les coutumiers monastiques sont, dans la plupart des cas, aussi peu utilisables que celles des textes liturgiques. Pourtant, à l'occasion, le liturgiste peut y glaner quelques précieux renseignements dont nous ferons état plus loin.

\section{Les témoignages des catalogues médiévaux}

Les catalogues médiévaux de bibliothèques s'avèrent d'une grande richesse, jusqu'ici presque entièrement inexploitée, dans le domaine des recherches sur la typologie des livres liturgiques médiévaux ${ }^{26}$. A n'en pas douter, une étude reste à faire sur le sens des désignations de l'ensemble des livres liturgiques dans ces catalogues. Dans le cadre plus restreint de cet article, nous les avons examinés dans une optique précise. Il s'agissait tout d'abord d'y détecter la moindre trace de libellus liturgique ; ensuite, de rassembler du matériel solide pour l'établissement d'une typologie ; enfin, de mieux cerner la nature codicologique et textuelle de ces documents ${ }^{27}$.

L'éventail lexical assez large que nous aurons à interpréter soulève des problèmes identiques à ceux déjà évoqués et que

24. Au IX ${ }^{e}$ siècle, Amalaire commente déjà ce passage, cf. Amalarii Episcopi opera omnia, t. II, Liber officialis, édité par J.-M. JANSSENS, Città del Vaticano, 1947 (Studi e Testi, 139), L. II, Ch. VIII/2.

25. C. VoGEL, R. ELZE, Le Pontifical romano-germanique du $\mathrm{X}^{e}$ siècle, t. I, Città del Vaticano, 1963 (Studi e Testi, 226), p. 17.

26. Sur l'intérêt de ce type de sources pour l'historien du Moyen Age, cf. A. Derolez, Les catalogues de bibliothèques, Turnhout, 1979 (Typologie des sources du Moyen Age occidental, fasc. 31).

27. Plusieurs catalogues que nous avons consultés ne figurent pas dans la liste qui va suivre, car aucune mention de libelli n'y apparaissait. Dans sa thèse sur les pontificaux du haut Moyen Age, N.K. Rasmussen avait sondé, on le verra, de façon non exhaustive, les catalogues publiés par G. Becker, à la recherche de libelli ; op. cit. en note 8 , t. III, p. $433-434$. 
François Dolbeau a partiellement démêlés ${ }^{28}$. Deux problèmes màjeurs se sont rapidement avérés délicats. La plupart des termes imprécis ont été éliminés, et seuls ont été retenus les termes explicites très variés, plus certains cas douteux mais pour lesquels on peut risquer une interprétation. Le second problème résidait dans l'évolution certaine à travers les siècles d'une même désignation. Là encore, une sélection stricte a été opérée, ne retenant, en priorité, que les mentions incluses dans des catalogues établis jusqu'au XIIe siècle compris. Quelques mentions postérieures méritaient toutefois d'être relevées, même si l'on ne peut être certain qu'elles désignent des documents plus anciens que l'époque de la rédaction du catalogue.

A côté des catalogues de bibliothèques, nous avons tiré un grand profit des inventaires de trésors médiévaux d'abbayes allemandes, édités par Bernhard Bischoff ${ }^{29}$, dont quelques passages figurent parmi les textes les plus intéressants pour notre propos.

Les résultats de l'enquête sont présentés à partir des différents termes retenus et en respectant, pour chaque mot, l'ordre chronologique.

\section{Libellus $\mathbf{s}^{30}$}

IXe siècle

Testament d'Ewrard, gendre de Louis le Pieux et marquis de Frioul, mort en 804, comprenant une liste de ses livres parmi lesquels on relève un " Librum orationum cum psalmis et libellum de orationibus $»$. Il s'agit vraisemblablement d'un petit livret de prières destiné à la dévotion privée dEwrard ${ }^{31}$.

Catalogue de l'abbaye de Reichenau établi au cours de l'abattiat de Régimbert (835-842) : «Libello continentur cantus gradualis et nocturnalis », petit livret contenant des pièces de chant de la messe et de l'office ; «Libello continentur orationes sufficientes ad sepulturam fratrum et orationes per singulas officinas monasterii et lectiones vel tractatus ad vigilias defunctorum recitandas »32, libellus réservé à la célébration des funérailles, à la bénédiction des lieux monastiques ainsi que des lectures pour l'office des morts.

28. Cf. supra, p. 14, F. DolbEAU, art. cit. en note 20.

29. B. BISCHOFF, Mittelalterliche Schatzverzeichnisse, I, Von der Zeit Karls des Grossen bis zur Mitte des 13. Jahrhunderts, München, 1967.

30. Sur les différents sens de ce terme, cf. F. DolbEAU, art. cit. en note 20, p. 9394 ; P. LEHMANN, « Mittelalterliche Büchertitel », dans Erforschung des Mittelalters, II, Stuttgart, 1962, p. 2.

31. Cf. P. RICHE, « Les bibliothèques de trois aristocrates laïcs carolingiens », dans Le Moyen Age, t. 69, 1963, p. 96-101, surtout note 60, p. 98.

32. Mittelalterliche Bibliothekskataloge, cité désormais $M B K$, Deutschlands und der Schweiz, I, München, 1918, p. 260, 1. 32 et p. 261, 1. 3-6. 


\section{$\mathrm{X}^{\mathrm{e}}$ siècle}

Catalogue de Sainte-Marie-Majeure de Crémone, 984 : « Episcopalium benedictionum libelli auro inscripti volumen unum ", libelli contenant des bénédictions épiscopales (reliés en un seul volume ?) ${ }^{33}$.

\section{XI' siècle}

Inventaire du trésor de l'abbaye de Pfävers, réalisé du temps de l'abbé Hartmann (av. 1030) ; la dernière mention dans la liste des livres liturgiques est : «I libellus cum tabulis elefantinis ${ }^{34}$; il est difficile d'imaginer un simple libellus protégé par une reliure d'ivoire, à moins qu'il ne s'agisse d'un volume beaucoup plus important que ne le laisse croire le terme, comme cela arrive pour des manuscrits non liturgiques ${ }^{35}$.

Catalogue de l'abbaye de Schaffhausen, 1083-1096; dans la liste des livres liturgiques, on peut lire : "Item alii libelli, quae (sic) in choro habentur », probablement des livrets de chant contenant les pièces nécessaires à une seule fête (messe ou office ?), ou peut-être tout autre espèce de libellus ${ }^{36}$.

\section{XII' siècle}

Liste de manuscrits légués par l'abbé Gotfrid (1162-1163) à l'abbaye SaintEmmeram de Ratisbonne : "Libellum de actione missarum "37; cette appellation désigne sans aucun doute l'Expositio missae de Florus de Lyon; bien qu'il ne s'agisse pas d'un livre liturgique, il est intéressant de le mentionner puisqu'il touche à des questions de liturgie.

Catalogue de Sant' Angelo in Formis, XII' siècle ; dans la liste des livres liturgiques, on lit : «Libella (sic) II »38, sans plus de précision.

Inventaire du trésor de l'abbaye de Prüfening, établi en 1165 : « Habemus missales XII cim preter missale cottidianum I principalis altaris et evangeliarium cum lectionario et preter collectarium festivum et libellos continentes missam pro defunctis et librum benedictionum ${ }^{39}$; à nouveau des petits livrets pour la messe des morts.

$\mathrm{XIII} \mathrm{I}^{\mathrm{e}} \mathrm{XV} \mathrm{V}^{\mathrm{e}}$ siècles (étant donné les datations des mentions qui suivent, on ne peut être assuré de l'ancienneté des manuscrits cités) :

Catalogue de l'abbaye de Kremsmünster, après 1316 : « Libellum missale 40 ; il peut s'agir d'un libellus missae avec un ou des formulaires de messes complets (oraisons, lectures, pièces de chants).

Catalogue de l'abbaye de Murbach, fin du XIVe siècle : " duo libelli cum oracionibus », probablement des livrets de prières ${ }^{41}$.

33. G. BECKER, Catalogi Bibliothecarum Antiqui, Bonn, 1885, $\mathrm{n}^{\circ} 36, \mathrm{p} .81$.

34. B. BISCHOFF, op. cit. en note $29, n^{\circ} 68$, p. 75.

35. Comme pour le ms Paris, Bibl. nat., lat. 1979, du IX ${ }^{e}$ siècle, composé de 251 folios et que son copiste appelle libellus, cf. F. DOLBEAU, art. cit. en note 20 , p. 93.

36. BECKER, $\mathrm{n}^{\circ} 93$, p. 157.

37. $M B K, \mathrm{IV} / 1,1977$, p. $151,1.22-23$.

38. BECKER, $\pi^{\circ} 120$, p. 83-84.

39. B. BisCHOFF, op. cit. en note $29, \mathrm{n}^{\circ} 73, \mathrm{p} .78$.

40. $M B K, \mathrm{~V}, 1971, \mathrm{p} .44,1.4$.

41. $M B K$, I, Österreich, 1915 , p. $136,1.30$. 
Catalogue de l'abbaye d'Admont, dans le diocèse de Salzbourg, établi en 1376 par Peter von Arbon : «Libellus sacramentorum 42 .

Liste des livres de l'évêque de Chiemsee, Iohannes Ebsers, dressée en 1445 : « Libellus continens capitula et collectas maiorum festivitatum per circulum anni (un collectaire festif); Libellus de cantu pertinente ad officia pontificalia cum consecrationibus et benedicionibus sacrorum vasorum et vestium ${ }^{43}$, livret noté contenant la section du pontifical pour la consécration des vases sacrés et la bénédiction des vêtements liturgiques.

\section{Quaternio}

Unité de base du manuscrit médiéval ${ }^{44}$, le quaternion désigne un cahier de huit feuillets et équivaut très souvent au libellus, surtout lorsqu'il n'est pas accompagné d'un chiffre, auquel cas il s'agit d'une liasse de cahiers non reliés ${ }^{45}$.

\section{IX ${ }^{\mathrm{e}}$ siècle}

Catalogue de l'abbaye de Saint-Gall : «Ordo romanus in duobus quaternionibus ${ }^{46}$, un libellus, de deux cahiers, contenant les textes d'une action liturgique non précisée ; puis «Orationes in quaternionibus ${ }^{47}$, s'agit-il d'un libellus missae ou bien d'un recueil d'oraisons?

Catalogue de l'abbaye de Lorsch : " Transitus sanctae Mariae in uno quaternione », libellus hagiographique de la fête de l'Assomption, ce n'est donc pas un libellus liturgique stricto sensu mais son rapport à la liturgie est certain ; "Orationes diversae in sex quaternionibus », recueil liturgique de caractère incertain $^{48}$.

\section{$\mathrm{X}^{\mathrm{e}}$-XII' ${ }^{\mathrm{e}}$ sièccles}

Catalogue de l'abbaye de Gorze (XI' siècle), où on lit en dernière mention : «Qualiter vel presbyter se praeparet ad missa celebranda in quaterniunculis scriptum ${ }^{49}$, prières préparatoires à la messe ; incluent-elles un ordo missae?

Catalogue de la bibliothèque épiscopale de Vich ; parmi les livres liturgiques, on lit: "Quaternos cum letania. Quaternos I cum letania et duos de

42. $M B K$, III, 1961, p. $57,1.3$.

43. MBK, IV, Österreich/Salzburg, 1966, p. 8, 1. 1-4.

44. Cf. W. WATTENBACH, Das Schriftwesen im Mittelalter, Leipzig, 1896, p. 176-179, et, plus récemment, L. J. Batalllon, « Exemplar, Pecia, Quaternus ", dans Vocabulaire du livre et de l'écriture, op. cit. en note 20, p. 208-211.

45. Cf. F. DolbEAU, art. cit. en note 20, p. 96.

46. BECKER, $\mathrm{n}^{\circ} 22$, p. 51 .

47. $M B K$, I, 1918 , p. $71,1.30$.

48. BECKER, $n^{\circ} 508$, p. 113 et $n^{\circ} 535$, p. 115 ; sur le catalogue de l'abbaye de Lorsch, cf. B. BISCHOFF, Die Abtei Lorsch im Spiegel ihrer Handschriften, $2^{\complement}$ édition, Lorsch, 1989, p. 18-28.

49. Cf. A. WAGNER, « Les manuscrits de la bibliothèque de Gorze. Remarques à propos du catalogue $»$, dans Religion et culture autour de l'An Mil. Royaume capétien et Lotharingie, D. IoGNA-PRAT, J.-Ch. PICARD éd., Paris, 1990, p. 111-117, spéc. p. 114 ; voir aussi G. MORIN, « Le catalogue des manuscrits de l'abbaye de Gorze au $\mathrm{XI}^{\mathrm{e}}$ siècle », dans Revue Bénédictine, t. 22, 1905, p. 1-11. 
dedicatione et quaternos de dedicatione ... quaterniones $I I \gtrsim^{50}$, on y reconnaît facilement des libelli servant à la dédicace d'une église, les autres sont plus difficiles à identifier. Nous ne connaissons pour notre part aucun libellus contenant seulement des litanies.

Catalogue de la bibliothèque de la cathédrale de Rouen (XII ${ }^{e}$ siècle) : "Orationes per annum in quaternis unius voluminis $\$ 1$, peut-être des recueils de libelli, soit divers formulaires de messes, soit des éléments de collectaire.

Catalogue de la bibliothèque du prieuré de Saint-Martin de la Canourgue, en Gévaudan (XI' siècle) : «Caterniones (sic) de antiphonas processionales ${ }^{52}$.

Catalogue de la bibliothèque de Saint-Emmeram de Ratisbonne, rédigé sous l'abbé Gotfrid (1162-1163) : "Orationem dominicam in uno quaternione »53 ; caractère impossible à préciser.

\section{Ordo}

- Les mentions « ordo romanus » ou « ordo » apparaissent très fréquemment dans les catalogues de bibliothèques du $\mathrm{IX}^{e}$ au XV $\mathrm{XV}^{e}$ siècle. Entités souvent indépendantes dans la liste des manuscrits liturgiques, elles désignent des libelli contenant tout ce qui est nécessaire à un seul rite liturgique, le plus souvent réservé à l'évêque, et n'apportent que rarement des indications précises sur le contenu. Voici une sélection des mentions les plus anciennes ou de celles qui nous semblent avoir le plus d'intérêt.

Catalogue de la bibliothèque de Saint-Gall, IX ${ }^{\mathrm{e}}$ siècle : « Ordo baptizandi $\aleph^{54}$, libellus du baptême.

Catalogue de la bibliothèque de la cathédrale de Salzbourg, avant 990 : «Ordo de katezizandis rudibus (sic) ${ }^{55}$.

Inventaire des cadeaux faits par l'évêque Abraham de Freising (957-994) au trésor de l'église de Maria Wörth près de Klagenfurt : «Ordo ad missam celebrandam 56 .

Catalogue de l'église de Saint-Martin de Tournai, XII siècle : «Benedictiones et ordo episcopalis ... ordo pontificalis ${ }^{57}$; il s'agit là, plus probablement, d'un bénédictionnaire et d'un pontifical.

Inventaire des livres et des objets liturgiques de la cathédrale de Bamberg, deuxième quart du $\mathrm{XI}^{\mathrm{e}}$ siècle : «Ordo I argenteus »58, puisque l'on se trouve dans un trésor de cathédrale, la reliure de ce document (s'agit-il d'un libellus ? ou plus probablement d'un pontifical) devait être précieuse.

50. E. JunYenT, « La Biblioteca de la Canónica de Vich en los siglos X-XI », dans Spanische Forschungen der Görresgesellschaft, I/2, Münster, 1963, p. 136145 , spéc. p. $139-140$.

51. BECKER, $\mathrm{n}^{\circ} 106$, p. 225.

52. L. DeLISLE, Le Cabinet des manuscrits de la Bibliothèque nationale, II, Paris, $1874, \mathrm{n}^{\circ} 35$, p. 506.

53. $M B K, \mathrm{IV} / 1,1977$, p. $151,1.25-26$.

54. BECKER, $\mathrm{n}^{\circ} 22$, p. 50.

55. Peut-être une allusion au texte de saint Augustin ; $M B K, \mathrm{IV}, 1966$, p. 18 , 1. 36-37.

56. B. BISCHOFF, op. cit. en note 29, p. 57.

57. Cf. L. DeLISLE, op. cit. en note 52, $\mathrm{n}^{\circ} 134$, p. 500 et $\mathrm{n}^{\circ}$ 286, p. 503.

58. B. BisCHOFF, op. cit. en note $29, \mathrm{n}^{\circ} 7$, p. 20. 
Catalogue de la bibliothèque de l'abbaye de Melk, $1483:$ "Ordo unguendi infirmum fratrem ... ordo ad communicandum infirmum ... ordo ad benedicendi mensam ${ }^{59}$; étant donné la date de ce catalogue, on ne peut savoir s'il s'agit de pièces anciennes mais, quoi qu'il en soit, ces libelli ont l'intérêt de préciser l'acte liturgique pour lequel ils doivent servir (onction et communion des frères malades, prières du repas).

\section{Termes divers 60}

Catalogue de la bibliothèque de Saint-Gall, IX siècle : « De nativitate sanctae Mariae in scedula "61, peut-être un livret de la fête de la Nativité de la Vierge, de facture modeste (ou une feuille volante), supplémentant un livre déjà existant.

Catalogue de la bibliothèque de la cathédrale de Crémone, 984 : "Defunctorum ordinis volumen unum » (des libelli pour les funérailles ?) ${ }^{62}$.

Catalogue de la bibliothèque de Saint-Amand, $\mathrm{XH}^{\mathbf{e}}$ siècle : «Volumen in quo continentur de diversis scripturarum voluminibus, et opusculum de actione missarum, et Rabanus de naturis rerum ... Officiorum mortuorum ${ }^{63}$, un recueil incluant l'expositio missae de Florus de Lyon et un libellus avec l'office des défunts.

Qu'apprenons-nous de toutes ces mentions sur le contenu, l'aspect codicologique, et donc la typologie du libellus liturgique?

En premier lieu, il faut noter que les hommes du Moyen Age ne semblent pas avoir fait un usage immodéré du terme même de libellus; ordo et quaternio apparaissent aussi souvent dans les catalogues pour désigner ce qui ressemble à des libelli.

Du point de vue codicologique, les deux derniers mots, surtout quaternio, fournissent des indications assez précises sur l'aspect matériel du document. Dans ce domaine, libellus apparaît beaucoup plus comme une désignation « livresque » dont on ne sait presque rien sur la matérialité.

Si l'on se penche maintenant sur les indications de contenu, on constate, en revanche, qu'est souvent clairement indiqué ce que contiennent les libelli désignés comme tels, alors que le quaternio perd ici toute la précision (sauf à une seule reprise avec les libelli de dédicace d'église) qu'il offrait dans le domaine codicologique. Pourtant, on spécifie bien que tant de quaternions contiennent des oraisons, mais sans donner de renseignement sur la ou les fêtes

59. $M B K$, Österreich, I, 1915 , p. 165 , 1. 10-11, p. $258,1.3$, p. $217,1.18$.

60 . Les termes d'agenda, baptisteria et obsequium, que l'on rencontre fréquemment dans les catalogues médiévaux, désignent le plus souvent des rituels plus complets que l'on indique par l'acte liturgique dont le texte est placé en tête du manuscrit ; ce ne sont pas à proprement parler des libelli.

61. BECKER, $\mathrm{n}^{\circ} 22$, p. 51 . Sur le sens du mot schedula, cf. F. DolBEAU, art. cit. en note 20, p. 96.

62. BECKER, $\mathrm{n}^{\circ} 36$, p. 81.

63. L. DeLISLE, op. cit. en note $52, n^{\circ} 361$, p. 472 et $n^{\circ} 27$, p. 484. 
pour lesquelles elles sont en usage. Les libelli désignés par ordo apparaissent, eux aussi, accompagnés à maintes reprises d'indications de contenu, surtout lorsqu'il s'agit du baptême, acte liturgique non réservé à l'évêque dont les rites spécifiques sont plutôt désignés par les mentions ordo romanus, ordo episcopalis, ordo pontificalis.

Les informations codicologiques de certaines mentions nous apprennent également que les libelli peuvent aussi bien être constitués d'un seul quaternion, voire d'un seul feuillet, que de plusieurs cahiers pouvant aller jusqu'à six quaternions. Cette grande diversité codicologique met bien en relief le point fondamental de la définition du libellus liturgique du haut Moyen Age : son contenu. Quelles que soient les différentes fonctions du libellus, diffusion du texte d'une fête ou bien nécessité de transporter un petit document pour un acte liturgique itinérant (entre autres), celui-ci se définit avant tout par son unité textuelle et liturgique, aussi bien pour le matériel euchologique (l'ensemble des oraisons) que pour les pièces de chant ou la description de rites (ordo). L'action liturgique pour laquelle le libellus est conçu détermine son contenu autant que sa présentation matérielle. Les différents cas qui vont être à présent passés en revue confirmeront de façon particulièrement nette les remarques et les conclusions énoncées à l'issue de l'examen des mentions de catalogues médiévaux.

Mais, avant de passer aux manuscrits, voyons rapidement les principales actions liturgiques attachées aux libelli recensés dans les catalogues. Beaucoup d'oraisons, recueils ou formulaires pour des fêtes particulières, souvent non précisées ; certains sont sans aucun doute des libelli missarum, d'autres mentions font plutôt penser au texte du canon de la messe, et même à un ordo missae; très peu de libelli destinés au chant, aucune mention de libelli réservés aux lectures, quelques fêtes particulières bien spécifiées (notamment en l'honneur de la Vierge) ; enfin, et surtout, des ordines ou des formulaires de messes pour les funérailles, l'onction des malades, le baptême et divers rites, comme la dédicace d'une église, réservés aux fonctions épiscopales. Nous verrons que cette liste se retrouve dans les libelli conservés et qu'elle s'explique par des pratiques liturgiques très spécifiques du haut Moyen Age.

\section{Le témoignage des manuscrits}

A cause de son aspect matériel, le libellus est forcément plus fragile que d'autres manuscrits. L'absence de reliure explique en partie que les libelli, liturgiques ou autres, se soient dans l'ensemble moins bien conservés que d'autres livres. Ils ont été 
sans aucun doute usés, abîmés, et surtout perdus plus facilement, ce qui laisse supposer qu'il en a existé une quantité beaucoup plus importante que le nombre, tout de même significatif, parvenu jusqu'à nous.

Dès le Moyen Age, on a constitué des recueils factices de libelli, certes sans grande unité interne, mais évitant ainsi qu'ils ne disparaissent totalement. Au XIII e siècle, à Saint-Martial de Limoges, Bernard Itier semble avoir eu le souci de relier ensemble des libelli de tout genre 64 . Une mention du catalogue de la bibliothèque de Muri, au XII siècle, précise : "Sunt adhuc hic opuscula libellorum satis utilia, que oportet servare et meliorare et non destruere quia nos non potuimus ea hic sigillatim describere ${ }^{65}$. A côté des recueils factices, beaucoup de libelli ont été insérés, et donc conservés, dans des recueils de textes divers constitués aux XIII', XIVe, $\mathrm{XV}^{e}$ siècles, ou à l'époque moderne. D'autres ont survécu grâce à leur fonction d'origine. C'est le cas de ceux, par exemple, qui sont venus enrichir le contenu liturgique d'un manuscrit déjà existant.

Les libelli présentés dans les pages qui suivent illustrent ces divers cas de conservation. La liste des documents ne prétend pas à l'exhaustivité. Elle a été établie à partir d'études de cas qui ont la plupart fait l'objet d'une publication, ainsi que de quelques répertoires de manuscrits liturgiques, principalement celui de Klaus Gamber, dont on sait qu'il est à manier avec précaution ${ }^{66}$. Seuls les plus représentatifs des différents types de libelli rencontrés au cours de cette enquête feront l'objet d'une brève description. D'autres ne seront signalés qu'à titre d'exemple pour tel ou tel type. Enfin, le choix s'est porté sur les manuscrits dont la nature de libellus est établie avec certitude, soit par notre examen personnel, soit par une publication sérieuse et approfondie ${ }^{67}$.

Le premier cas de figure concerne les libelli qui ont servi à la diffusion du texte d'une ou de plusieurs fêtes particulières. C'est le

64. Comme il l'indique lui-même dans le ms Paris, Bibl. nat., lat. 2770, au fol. 179 : «Anno 1205 fecit me ligare Bernardus Iterii armarius, et quatuor quaterniones ultimos qui antea non erant mecum adiunxit »; le dernier quatemion est un libellus liturgique de l'office de saint Jean-Baptiste, du XII ${ }^{e}$ siècle.

65. BECKER, $n^{\circ} 122$, p. 252.

66. K. GAMBER, Codices Liturgici Latini Antiquiores, 2 vol., Fribourg, 1963 et 1968, plus un volume de supplément, Fribourg, 1988 (cité désormais $C L L A$ ).

67. D'emblée, ont été exclus tous les manuscrits que certains auteurs ont identifiés comme des libelli, mais qui se sont avérés être des fragments de manuscrits plutôt que des libelli. A cet égard, le cas le plus représentatif est celui du ms lat. 958 de la Bibliothèque nationale de Vienne que Gamber nomme libellus (CLLA, $\mathrm{n}^{\circ} 764$, p. 537), mais qui est en réalité un fragment de sacramentaire, peut-être jamais entièrement réalisé ; voir à ce propos les mises au point de N. K. RASMUSSEN, op. cit. en note 8, t. II, p. 358-359 et de F. UNTERKIRCHER, Karolingisches Sakramentar (Codex Vindobonensis 958), Graz, 1971 (Codices selecti, 25). 
moment de rappeler les deux lettres adressées aux moines de SaintVaast d'Arras et de Fulda dans lesquelles Alcuin leur annonce l'envoi de formulaires de messes votives qu'il a lui-même composées ${ }^{68}$. Les termes mêmes utilisés par Alcuin dans les lettres, ainsi que les libelli de messes votives conservés (qui ne sont pas ceux envoyés par le maître carolingien), permettent de penser que des libelli ont accompagné les lettres. Les libelli de l'office et de la fête de la Toussaint contenus dans un manuscrit de Munich, Bayerische Staatsbibliothek, Clm 14704 (fol. 121-144) ${ }^{69}$, réalisé à Freising sous l'épiscopat d'Anno (854-875), illustrent ce cas de figure. D'autres témoins du IXe siècle, comme le manuscrit de Rouen, Bibl. mun., A. 566 (275), sur lequel nous reviendrons à propos des libelli missarum, attestent cette pratique. Ce doit être également le cas pour un manuscrit, vraisemblablement du $\mathrm{XI}^{\mathrm{e}}$ siècle, de la bibliothèque capitulaire de Tolède (cod. 35.7) qui, sur 54 folios, contient l'ensemble des textes nécessaires à certaines fêtes de la Vierge (Assomption et Conception), plus un office de l'Assomption ${ }^{70}$. Cette façon de procéder pour diffuser les textes de fêtes précises a perduré pendant une bonne partie du Moyen Age. Le ms 3719 du fonds latin de la Bibliothèque nationale conserve deux libelli contenant plusieurs offices votifs dédiés à la Vierge (deux quaternions, fol. 93-100 et 101-108) 71 $^{\text {. Vraisemblablement }}$ réalisés dans le sud-ouest de la France à la fin du XI'e siècle, ces deux libelli furent jadis pliés en quatre, à l'instar de certains libelli hagiographiques. Ces traces témoignent de la façon dont on les a transportés. Citons encore les cinq cahiers du ms lat. 1143 de la Bibliothèque nationale, office et messe du Corpus Christi composés

68. Cf. supra, p. 14 et note 22 ; sur l'activité liturgique d'Alcuin, voir aussi l'article très documenté de J. DEshusses et de H. BARRE, « A la recherche du missel d'Alcuin », dans Ephemerides Liturgicae, t. 82, 1968, p. 3-44.

69. Cf. B. BIscHoFF, Die südostdeutschen Schreibschulen und Bibliotheken in der Karolingerzeit, I, Wiesbaden, 1960, p. 126.

70. $C L L A, \mathrm{n}^{\circ} 320$, p. 203 ; J. JANINI, Catálogo de los manuscritos litúrgicos de la catedral de Toledo, Toledo, 1977, p. 103-104. Si l'on se réfere aux descriptions données par certains auteurs, d'autres témoins hispaniques du $\mathrm{XI}^{\mathbf{e}}$ siècle pourraient être des libelli servant à la diffusion de la fête d'un saint, comme par exemple dans les manuscrits, Madrid, Academia de la Historia, cod. Aemilian. 60 (fol. 42-48v), fête des saints Cosme et Damien (cf. J. JANINI, Manuscritos litúrgicos de las bibliotecas de España, I, Burgos, 1977, p. 162 ; CLLA, n 321, p. 203) ; Paris, Bibl. nat., n.a.1. 239 (fol. 68-83), vraisemblablement copié à Silos au $\mathrm{X}^{\mathbf{e}}$ siècle, après 966 , qui contient l'office et la messe de sainte Pélagie mêlés à la passion de la sainte, cf. l'article de G. PHILIPpart, dans Analecta Bollandiana, t. 92, 1974, p. 70, note 1 et Bibliothèque nationale, C.R.M.E., Manuscrits enluminés de la péninsule ibérique, Paris, 1982, p. 9-10.

71. Catalogue général des manuscrits latins de la Bibliothèque nationale, VI, Paris, 1975, p. 578-589 ; les pièces du premier libellus, parmi lesquelles se trouve un des plus anciens témoins du Salve Regina noté (fol. 99v-100), ont été éditées par J.-M. CANAL, dans Ephemerides Mariologicae, t. 11, 1961, p. 506-509. 
par saint Thomas d'Aquin, qui ont servi à la fin du XIII' siècle à la diffusion de la fête nouvellement instituée ${ }^{72}$.

Avant d'aborder les libelli les plus révélateurs de différentes pratiques de la liturgie médiévale, passons rapidement en revue un type relativement fréquent, celui du libellus venant enrichir le contenu d'un manuscrit préexistant. Le sacramentaire de Nonantola (Paris, Bibl. nat., lat. 2292), réalisé dans la seconde moitié du IX siècle dans l'entourage de Charles le Chauve, fut complété au $\mathrm{XI}^{\mathrm{e}}$ siècle, à Nonantola même, avec un cahier de six feuillets, placé à la fin du manuscrit, qui contient le texte d'un ordo pour la dédicace d'une église. M. Gros a montré que l'écriture de cet ordo (écriture italienne), du XIe siècle, ainsi que son texte indépendant de la tradition romano-gallicane, font penser que ce libellus fut composé pour adapter le manuscrit carolingien à la liturgie locale ${ }^{73}$. Un peu plus tard dans le Moyen Age, un cahier inséré dans le ms Paris, Bibl. nat., lat. 933 (fol. 155-162v) témoigne encore de cette pratique. Il s'agit d'un collectaire-rituel de l'abbaye de Lagrasse, de la seconde moitié du XI' siècle, auquel on a ajouté, au XII' siècle, un libellus de la dédicace de l'église, peut-être à l'occasion d'une visite papale $^{74}$. D'autres témoins de libelli de dédicace ont subsisté, mais ne sont pas forcément insérés dans un manuscrit liturgique. Les quatre feuillets, du XI ${ }^{\mathrm{e}}$ siècle, ajoutés à la fin du manuscrit de la bibliothèque capitulaire de Lucques (cod. 605), contenant l'ordo ambrosien pour la dédicace, en fournissent un bon exemple. Rien ne prouve que ce libellus ne fut pas à un moment ou un autre inséré dans un manuscrit liturgique, ou qu'il n'a pas circulé ou servi de façon indépendante ${ }^{75}$.

Avant de parvenir à une forme complète et définitive, le missel passa par des expériences préliminaires qui peuvent être considérées

72. Sur cette fête et la part prise par Thomas d'Aquin dans la composition de ces textes liturgiques, cf. P.-M. GY, « L'office du Corpus Christi et saint Thomas d'Aquin », dans Revue des Sciences philosophiques et théologiques, t. 64, 1980, p. 491-507.

73. M. Gros, «L'ordo pour la dédicace des églises dans le sacramentaire de Nonantola », dans Revue Bénédictine, t. 79, 1969, p. 368-374.

74. Il s'agit de l'ordo du pontifical romain du XII ${ }^{e}$ siècle, cf. M. ANDRIEU, $L e$ pontifical romain au Moyen Age, I, Città del Vaticano, 1938 (Studi e Testi, 86), p. 176-195. Sur le manuscrit, cf. P.-M. GY, art. cit. en note 11, p. 454 et 456 ; Catalogue général des manuscrits latins de la Bibliothèque nationale, I, Paris, 1939, p. 330-331 ; selon Marie-Thérèse Gousset, que je remercie vivement pour ses indications, ce libellus fut probablement écrit par un scribe italien travaillant dans le sud-ouest de la France.

75. Sur ce libellus, cf. CLLA, $\mathrm{n}^{\circ} 575$, p. 281 ; P. BoRELLA, « L'ordo ambrosiano de G. Mercati per la dedicazione della chiesa », dans Ephemerides Liturgicae, t. 72, 1958 , p. $48-50$; N. K. RASMUSSEN, "Unité et diversité des pontificaux latins aux $\mathrm{VIII}^{\mathrm{e}}, \mathrm{IX}^{\mathrm{e}}$ et $\mathrm{X}^{\mathrm{e}}$ siècles », dans Liturgie de l'Église particulière et liturgie de l'Église universelle, Conférences Saint-Serge, XXI' Semaine d'études liturgiques, Rome, 1976 , p. 401. 
comme des formes embryonnaires : ce sont les libelli missarum. Composés de quelques cahiers, allant de un à quatre ou cinq, ils contiennent les textes pour une seule ou plusieurs fêtes. La structure des formulaires comprend à la fois les oraisons (propres au sacramentaire), les lectures, normalement insérées dans l'évangéliaire ou le lectionnaire, et les incipit des pièces de chant contenues dans le graduel. Tout le matériel nécessaire au déroulement de la célébration étant réuni, le prêtre est ainsi équipé d'un petit livret lui permettant de célébrer seul, s'il le désire.

Ce type de libellus missae fut vraisemblablement très utilisé en milieu monastique, en particulier pour la célébration des messes privées, pratique liturgique sur laquelle nous reviendrons. Le ms 610 de la bibliothèque de l'Arsenal à Paris (Reichenau, fin du $\mathrm{X}^{\mathrm{e}}$ siècle) a été conçu et réalisé précisément pour la célébration de messes privées. Sur 62 folios, le manuscrit ne contient, en plus du canon de la messe, que quatre messes votives (Sainte Trinité, pour des nécessités diverses, Sainte Croix, messe pour les défunts) comprenant les oraisons, les pièces de chant et les lectures. La nature très particulière de ce codex, surtout le gonflement de chaque formulaire des quatre messes par une multitude d'oraisons, autorise l'hypothèse qu'il s'agit là d'une solution pratique apportée au problème de la multiplication des messes privées au Moyen Age. Au lieu de célébrer successivement plusieurs messes dans leur intégralité, le moine-prêtre aurait utilisé ce libellus missae qui lui permettait de les célébrer, en une seule fois, en se limitant au canon et aux nombreuses oraisons transcrites dans le manuscrit ${ }^{76}$.

Tous les libelli missarum n'ont pas servi à ce genre de pratique liturgique. Un grand nombre d'entre eux ont permis la diffusion d'une fête particulière, mais, à la différence des libelli de ce type dont on a déjà parlé, ils contiennent non seulement les oraisons, mais aussi les lectures et les pièces de chant. Le premier cahier de 8 folios du ms Rouen, Bibl. mun., A. 566 (275), présente les formulaires complets de sept messes votives en l'honneur de la Vierge et de tous les saints. Il fut réalisé au scriptorium de SaintAmand dans la seconde moitié du $\mathrm{IX}^{\mathrm{e}}$ siècle pour être envoyé aux

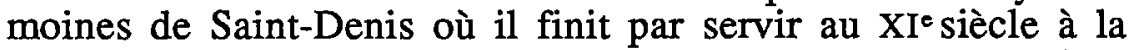
formation d'un petit missel dont le contenu est calqué sur celui du libellus missae de Saint-Amand ${ }^{77}$.

76. Cf. N. K. Rasmussen et E. Palazzo, « Messes privées, livre liturgique et architecture. A propos du ms Paris, Arsenal 610 et de l'église abbatiale de ReichenauMittelzell ", dans Revue des Sciences philosophiques et théologiques, t. 72, 1988, p. 77-87.

77. Cf. E. Palazzo, « Un libellus missae du scriptorium de Saint-Amand pour Saint-Denis. Son intérêt pour la typologie des manuscrits liturgiques ", dans Revue Bénédictine, t. 99, 1989, p. 286-292. 
D'autres libelli du même type apparaissent toutefois amputés des incipit des pièces de chant et sont toujours restés indépendants. C'est le cas pour le libellus missae palimpseste de Benediktbeuren, copié vers 800 , qui contient les messes de l'Ascension et de la Pentecôte (vigile et jour de la fête) (München, Bayerische Staatsbibliothek, $\mathrm{Clm} 6333,4$ fol. dispersés) ${ }^{78}$, ou encore pour celui, peut-être du VII' siècle et là aussi palimpseste, constitué de 44 feuillets formant six cahiers, avec les textes de six messes dominicales et une propre pour saint Germain (Karlsruhe, Badische Landesbibliothek, Aug. CCLIII) ${ }^{79}$.

Des formes plus restreintes de libelli de messes, toujours amputés des pièces de chant, subsistent à côté des libelli plus volumineux signalés précédemment. Le ms Reg. lat. 191 de la bibliothèque du Vatican est un recueil de textes divers, provenant de Saint-Remi de Reims, parmi lesquels on compte des parties liturgiques ${ }^{80}$. Les folios 54-62 (un quaternion), probablement écrits à Reims au $\mathrm{IX}^{\mathrm{e}}$ ou au $\mathrm{X}^{\mathrm{e}}$ siècle, contiennent les textes du canon de la messe ainsi que de quelques messes votives, notamment pour les malades et les défunts ; les lectures sont jointes à la fin du quaternion $^{81}$. Ce petit libellus a probablement servi dans le cadre limité de certaines actions liturgiques (onction des malades et messe des funérailles).

Le $\mathrm{P}$. Gy a récemment mis en garde, à propos de certains libelli, contre des appellations trop vite énoncées ${ }^{82}$. Dans bien des cas, des auteurs ont été trompés par la présence d'une section liturgique à l'intérieur d'un manuscrit contenant d'autres textes. L'homogénéité codicologique et paléographique de ces manuscrits (section

78. Cf. E. MundING, A. DolD, Benediktinische und liturgische Texte aus Clm 6333, Beuron, 1930, p. 56-60; CLLA, n 704, p. 331.

79. A. WILMART, "L'âge et l'ordre des messes de Mone », dans Revue Bénédictine, t. 28,1911 , p. 377-390;CLLA, $\mathrm{n}^{\circ} 203$, p. 158-159.

80. Cf. A. WilmarT, Codices Reginenses Latini, I, 1937, p. 452-458; P. SALMON, Les manuscrits liturgiques latins de la Bibliothèque Vaticane, t. III, Città del Vaticano, $1970, \mathrm{n}^{\circ} 217$, p. 75 et t. II, $1969, \mathrm{n}^{\circ} 27$, p. 18 ; F. DOLBEAU, «Un catalogue fragmentaire des manuscrits de Saint-Remi de Reims au XII ${ }^{e}$ siècle », dans Recherches Augustiniennes, vol. 23, 1988, p. 213-243, spéc. p. 216 et 224.

81. Les autres parties liturgiques de ce recueil sont sans lien paléographique avec ce cahier. Leurs contenus (fragment d'un ordo du baptême et rites de la pénitence et de la réconciliation) pourraient suggérer là aussi des libelli, mais leur état codicologique empêche toute conclusion à leur sujet. Les mêmes précautions sont à prendre pour d'autres manuscrits, rapidement nommés libellus, comme par exemple pour le ms Brescia, Biblioteca Queriniana, cod. G. VI 7 (CLLA, n 820 , p. 379); Saint-Gall, Stiftsbibliothek, cod. 150 (CLLA, $\mathrm{n}^{\circ} 894$, p. 404) ; Vatican, Palat. lat. 489 (CLLA, $\mathrm{n}^{\circ} 1683$, p. 608 ; SALMON, op. cit., I, 1968, p. 63) ; ou encore les fol. 1-6, 103 et 192v-201 du ms Paris, Bibl. nat., lat. 1603 qui sont en fait des fragments de sacramentaire du VII" siècle (CLLA, $\mathrm{n}^{\circ} 807$, p. 375, mais surtout $\mathrm{A}$. WILMART, «Les messes de la collection de Saint-Amand ", dans Jahrbuch für Liturgiewissenschaft, Bd 3, 1923, p. 67-77).

82. Cf. P.-M. GY, art cit. en note 13. 
liturgique comprise) invite à plus de prudence dans les conclusions. Souvent, ces sections liturgiques ne sont que des copies de probables libelli, comme par exemple les folios 87-104, rituel des funérailles avec le plus ancien témoin ambrosien de l'office des défunts, du ms Paris, Bibl. nat., lat. 2984 A (probablement de la fin du IX siècle), codicologiquement et paléographiquement homogène avec l'ensemble du manuscrit ${ }^{83}$.

Ce dernier exemple nous permet d'aborder un autre type de libellus : le livret destiné à une ou plusieurs actions liturgiques bien déterminées, autres que la célébration eucharistique pour laquelle sont conçus les libelli missarum. La grande majorité de ces libelli, véritables petits rituels selon la terminologie du P. Gy, ont été confectionnés pour servir aux rites de la pénitence, de l'onction des malades et des funérailles. Une telle spécialisation ne surprend pas. On verra, dans la dernière partie de cet article, le sort particulier réservé par les hommes du Moyen Age au déroulement de ces rites.

Mentionnons, pour le moment, quelques exemples significatifs. Tout d'abord, les folios 90-116v du ms Paris, Bibl. nat., lat. 13764, réalisé à Saint-Amand vers 900 , puis envoyé à SaintRemi de Reims et inséré aujourd'hui dans un recueil provenant de Reims ${ }^{84}$. Avec ses deux rituels, de la pénitence et de l'onction des malades (avec la messe pour les malades comprenant les lectures et les pièces de chant), il fut sans aucun doute destiné aux prêtres lors de visites rendues aux malades. Un rituel de l'onction des malades, un ordo des funérailles et un calendrier occupent les deux premiers cahiers d'un des recueils provenant de Saint-Martial de Limoges (Paris, Bibl. nat., ms lat 1240 , fol. 1-16, probablement du $\mathrm{X}^{\mathrm{e}}$ siècle) ${ }^{85}$. Ces textes ont servi, à l'état indépendant, pour le déroulement de ces rites ${ }^{86}$. Pour les funérailles, on peut citer aussi les fragments palimpsestes ( 2 fol., Italie du nord, VII ${ }^{e}$ siècle) de Stuttgart (Württembergische Landesbibliothek, cod. H. B. VII.

83. Cf. P.-M. GY, art. cit. en note 11, p. 457-458. D'autres cas de copies de libelli, interprétés comme tels, sont célèbres : Avignon, Bibl. mun., ms 175 (fol. 18) et Montpellier, Faculté de médecine, ms 12 (fol. 9), du IXe siècle, cf. A. WilMarT, «Un livret bénédictin composé à Gellone au commencement du $\mathrm{IX}^{\mathbf{e}}$ siècle », dans Revue Mabillon, t. 12, 1922, p. 119-133 et les mises au point apportées par N. K. RASMUSSEN, «Une Cartula missalis retrouvée », dans Ephemerides Liturgicae, t. 83, 1969, p. 482-484 ; Bruxelles, Bibliothèque Royale, ms 10027-44 (fol. 125135), VIII'-IX ${ }^{e}$ siècles, cf. CLLA, $\mathrm{n}^{\circ} 856$, p. 392 et P. SIFFRIN, dans Ephemerides Liturgicae, t. 45,1931 , p. $327-353$.

84. Cf. É. Palazzo, « Les deux rituels d'un libellus de Saint-Amand (Paris, Bibl. nat., lat. 13764) », dans Rituels. Mélanges offerts au père Gy, Paris, 1990, p. 423436.

85. Catalogue général des manuscrits latins de la Bibliothèque nationale, $\mathrm{I}$, Paris, 1939, p. 459.

86. Cf. P.-M. GY, art. cit. en note 11, p. 457. 
$10)^{87}$, et le libellus, plus récent ( $\mathrm{X}^{\mathrm{e}}$ siècle), du monastère de $S$. Eutizio in Valcastoriana en Ombrie (Rome, Biblioteca Vallicelliana, cod. C. 10, fol. 133-138), inséré aujourd'hui dans un évangéliaire du XIre siècle ${ }^{88}$. D'autres libelli ont uniquement servi dans le cadre de la liturgie pénitentielle. Un des exemples les plus représentatifs en est le ms du Vatican, Reg. lat. 207 (fol. 85-105), du IX' siècle $^{89}$.

Si les folios 1 à 3 du ms du Vatican, Reg. lat. 1000A (du Xe ou $\mathrm{du} X \mathrm{XI}^{\mathrm{e}}$ siècle) constituent bien un petit libellus, celui-ci représente une forme unique de petit rituel monastique, livret contenant les oraisons et les bénédictions pour les divers locaux monastiques, entre autres ${ }^{90}$.

Des recueils de bénédictions réservées à l'évêque et transcrites dans des libelli sont, de leur côté, bien attestés, comme en témoignent les folios 1-14 d'un manuscrit de Munich (Bayerische Staatsbibliothek, $\mathrm{Clm} 6430$, IX $\mathrm{Xu}^{\mathrm{e}} \mathrm{X}^{\mathrm{e}}$ siècle, Freising) ${ }^{91}$. Après les recherches déjà évoquées de N.K. Rasmussen en matière de libelli épiscopaux, il est inutile de revenir en détail sur ce type de libellus $^{22}$. Rappelons simplement que l'auteur a d'une part élaboré une typologie du document liturgique épiscopal, extrêmement fructueuse pour l'histoire de la pratique liturgique, notamment en ce qui concerne les déplacements de l'évêque ou, au contraire, la liturgie de la cathédrale. Il a d'autre part montré que certains pontificaux des $\mathrm{IX}^{\mathrm{c}}$ et $\mathrm{X}^{\mathrm{c}}$ siècles étaient en réalité des recueils factices de libelli à l'origine indépendants les uns des autres. Avant de former un pontifical à part entière, chaque libellus servait pour une action liturgique propre à l'évêque (ordinations ecclésiastiques, recueil de bénédictions, ordo pour un concile, consécration

87. $C L L A, \pi^{\circ} 650$, p. 316 ; K. GAMBER, « Das Stuttgarter SakramentarPalimpsestblatt aus Cod. H. B. VII. 10 », dans Archiv für Liturgiewissenschaft, Bd 6, 1960, p. 455-460.

88. CLLA, n ${ }^{\circ} 1025$, p. $436 ; \mathrm{R}$. AMIET, « Catalogue des livres liturgiques manuscrits conservés dans les archives et les bibliothèques de la ville de Rome », dans Studi Medievali, t. 27, 1986, p. 955.

89. Cf. SALMON, III, 1970, $\mathrm{n}^{\circ} 218$, p. 75 ; pour la liturgie pénitentielle, les ms du Vatican, lat. 1339 ( $\mathrm{X}^{\mathrm{e}}$ siècle, $C L L A, \mathrm{n}^{\circ} 1599$, p. 573 ; SALMON, III, $\mathrm{n}^{\circ} 260$, p. 83), lat. 5768 (SALMON, II, $n^{\circ} 408$, p. $158-159$ et III, $n^{\circ} 274$, p. 86), Chigi C.V. 134 ( $\mathrm{X}^{\mathrm{e}}$ siècle, SALMON, II, $\mathrm{n}^{\circ} 277$, p. 118) et Palat. lat. 485 (SALMON, II, p. 14-15) sont difficiles à interpréter à partir des descriptions faites par Salmon.

90. $C L L A, \mathrm{n}^{\circ} 865$, p. 97 ; SALMON, $\Pi, 1969, \mathrm{n}^{\circ} 33$, p. 21 , pense que l'on a affaire à un fragment de sacramentaire. L'antienne d'offertoire transcrite au fol. $3 \mathrm{v}$ est postérieure à l'écriture des bénédictions et des oraisons. Le manuscrit, originaire de Reims, est un recueil de canons. Pour l'identification des pièces, cf. K. GAMBER, S. REHLE, «Fragmenta liturgica », dans Sacris Erudiri, t. 23, 1978/1979, p. 305310.

91. CLLA, $\mathrm{n}^{\circ} 280$, p. 184 ; J. DESHUSSES, « Le bénédictionnaire gallican du VII' siècle », dans Ephemerides Liturgicae, t. 77, 1963, p. 169-187.

92. N. K. RASMUSSEN, op. cit. en note 8. 
d'église...). Leur compilation a permis ensuite de les conserver et de constituer la substance d'un nouveau livre liturgique : le pontifical ${ }^{93}$.

Les travaux déjà cités de M. Huglo, entre autres ${ }^{94}$, ne laissent aucun doute sur l'affirmation suivante : plusieurs livres de chant liturgique du haut Moyen Age résultent de la compilation de libelli qui contenaient les pièces pour telle ou telle fête. Quelques tropaires-prosaires des $\mathrm{X}^{\mathrm{c}}$ et XI $\mathrm{XI}^{\mathrm{e}}$ siècles en portent toujours les traces à travers leur organisation interne, alors que la plupart des livres de chant ont fait l'objet de remaniements qui ont supprimé les marques visibles de leur mode de constitution. Même si l'on peut supposer l'existence d'un grand nombre de libelli de chant, on n'en trouve plus aujourd'hui de preuves abondantes, ni dans les catalogues médiévaux, ni à travers les manuscrits conservés. Ceci s'explique probablement par la pratique même des chantres médiévaux. La nécessité pour ces derniers de constituer, ou d'utiliser, des recueils de pièces ayant circulé dans des libelli à l'état indépendant, a certainement favorisé la disparition progressive de livrets qui servaient avant tout à la diffusion de pièces propres à une fête.

Des raisons touchant à la pratique liturgique sont aussi à l'origine de l'absence presque totale, aussi bien dans les textes que dans les documents, de libelli réservés à la liturgie de la parole. Le Moyen Age n'a pour ainsi dire pas connu de libellus propre au diacre ou au sous-diacre, ou à un autre lecteur, comme c'était le cas pour le célébrant ou le chantre, notamment. On ne doit pas s'étonner de cette absence, car les lectures (évangiles ou autres textes) étaient transcrites dans le livre des évangiles avec Capitulare evangeliorum, dans l'évangéliaire, le lectionnaire, ou même dans des libelli missarum pour des fêtes particulières. On n'éprouvait pas le besoin de fabriquer des libelli pour les textes saints ; en effet, aucune création de nouveaux textes pour la lecture ne devait être diffusée, contrairement aux ordines, oraisons, pièces de chant, qu'il fallait faire connaître.

Seul le ms Aschaffenbourg, Hofbibliothek, 2, paraît constituer une sorte de libellus evangeliorum que la réalité liturgique caractérise plutôt comme un évangéliaire festif particulièrement restreint ${ }^{95}$. Réalisé à Fulda au cours du troisième quart du $\mathrm{X}^{\mathrm{c}}$ siècle, il s'agit d'un quinion (10 fol.) parfaitement homogène qui semble

93. Les trois principaux manuscrits, analysés par Rasmussen, pour lesquels on observe ce phénomène sont : Leyde, B.P.L., 111.2 (RASMUSSEN, ibid., t. I, p. 151186) ; Reims, Bibl. mun., ms 340 (t. II, p. 275-306) et Bibl. Vat., lat. 7701 (t. II, p. 307-336).

94. Cf. M. Huglo, art. cit. en notes 9 et 10.

95. Cf. J. Hofmann, H. ThuRn, Die Handschriften der Hofbibliothek Aschaffenburg, Aschaffenburg, 1978, p. 2-5. 
avoir toujours été indépendant ${ }^{96}$. Son contenu se limite à une série de péricopes évangéliques pour certaines grandes fêtes de l'année liturgique (Noël, Circoncision, Épiphanie, Purification, vigile et jour de Pâques, Ascension...). Son court sanctoral ne donne pas plus de renseignements sur son lieu d'utilisation ; la fête du saint patron de Fulda, Boniface, n'y a pas été retenue. Ce manuscrit, d'aspect somptueux, fut sans doute utilisé lors de grandes fêtes de l'année liturgique, en remplacement d'un évangéliaire normal ; pour notre propos, il constitue en tout cas un unicum.

La présentation de ces quelques libelli conservés appelle plusieurs remarques sur la typologie du libellus liturgique. En premier lieu, il faut relever la grande correspondance entre les mentions des catalogues médiévaux et les témoins manuscrits. Tant $\mathrm{du}$ point de vue codicologique que liturgique, les libelli parvenus jusqu'à nous semblent refléter la vision du libellus liturgique telle qu'elle se profile à travers les mentions des catalogues anciens. Cette constatation nous paraît aussi bien éclairer le travail du liturgiste que celui du spécialiste des catalogues médiévaux.

En second lieu, dans le vaste domaine de l'histoire des livres liturgiques, la confrontation de ces différents types de sources s'avère féconde pour une meilleure compréhension du libellus. Quel que soit son aspect codicologique, il se définit avant tout par son contenu liturgique. Les principaux types sont : le libellus missae (beaucoup de messes votives, avec pièces de chant ou non); le rituel (dans la majorité des cas, les funérailles, l'onction des malades, le viatique et la pénitence); le libellus épiscopal (pouvant aller de la dédicace d'église aux ordinations ecclésiastiques); et dans une moindre mesure le libellus de chant. Pour l'ensemble de ces différents types, il faut distinguer le libellus servant à diffuser le texte d'une fête (formulaire de messe ou ordo), de celui dont le seul but est de servir à l'exécution d'un rite particulier (rituels, ordines épiscopaux et recueils de bénédictions non spécifiques à l'évêque). Certains libelli remplissent les deux fonctions ; ce sont en particulier les libelli missarum, propres à la célébration eucharistique, notamment pour des messes votives. A côté du rôle fondamental qu'ils ont joué dans la formation de plusieurs livres liturgiques (missel, pontifical, entre autres), les différents types de libelli mis en évidence apparaissent également comme les témoins privilégiés de quelques-unes des pratiques liturgiques du haut Moyen Age.

96. Cf. E. PALAzzo, Les sacramentaires de Fulda : étude sur l'iconographie et la liturgie à l'époque ottonienne, Thèse de doctorat, université de Paris X-Nanterre, 1990 , t. II, p. 332-333. 


\section{Les libelli, témoins de la pratique liturgique}

N. K. Rasmussen a abordé de façon neuve les problèmes posés au liturgiste par les différents types de célébration du haut Moyen $\mathrm{Age}^{97}$. A partir de l'étude précise de plusieurs manuscrits médiévaux, l'auteur a essayé de systématiser une approche de ces derniers qui repose sur un principe simple : le contenu et l'aspect matériel d'un manuscrit renseignent sur son utilisation. Si bien qu'il existe autant de formes de célébration que de types de documents. Pour prendre un exemple simple, les sacramentaires ont aussi bien servi en milieu monastique, ou paroissial, que dans le cadre de la liturgie épiscopale ; pour chaque type de célébration, les formes externes et internes des manuscrits sont différentes.

N.K. Rasmussen affirmait alors : « les libelli sont toujours vivants $\gg 98$, et pensait que leur aspect modeste correspondait plutôt à des documents à usage presbytéral ; nous allons le voir, c'est en partie vrai. Mais avant de passer aux pratiques liturgiques du prêtre, ou du moine-prêtre, repérables à travers les libelli, arrêtons-nous brièvement sur les libelli épiscopaux dont N. K. Rasmussen a également défini une typologie ${ }^{99}$. Passant du libellus de voyage à celui propre à l'usage cathédral, l'auteur a ainsi retrouvé les témoins « vivants » des diverses formes de la célébration des rites par l'évêque. A propos des manuscrits, il faut observer que bon nombre de libelli épiscopaux, rassemblés ensuite en recueils factices, sont matériellement bien plus modestes que certains libelli à usage presbytéral ; car c'est en réalité l'usage que l'on fait d'un libellus (qu'il soit destiné au prêtre ou à l'évêque) qui détermine sa forme matérielle. Pour conclure avec les libelli épiscopaux, rappelons l'intérêt d'un passage du testament de l'évêque d'Elne en 915 , fait en faveur de son église : «... ad ecclesiam consecrandam quaterniones duos, ad visitandum infirmum quaterniones duos, ad ordinationes ecclesiasticos quaternione uno »100. Il s'agit là d'une description intéressante des désignations typologiques correspondant aux différentes pratiques liturgiques.

Lorsqu'on aborde la célébration presbytérale, les mondes monastique et paroissial se confondent assez rapidement. Le rôle joué, à côté des prêtres, par les moines au sein des paroisses, a été bien mis en lumière dans de nombreux travaux ${ }^{101}$. Pour notre

97. N. K. RASMUSSEN, "Célébration épiscopale et célébration presbytérale : un essai de typologie ", dans Segni e riti nella chiesa altomedievale occidentale, Settimane di studio del Centro Italiano di Studi sull'alto Medioevo, XXXIII, 11-17 avril 1985, Spoleto, 1987, t. I, p. 581-603.

98. Ibid., p. 588.

99. N. K. RASMUSSEN, op. cit. en note 8, surtout vol. III, p. 415 sq.

100. P.L., 132, col. 468.

101. Plusieurs raisons sont à l'origine de cette part active prise par les moines dans certaines paroisses : évangélisation, possession des terres par les monastères, 
propos, il est intéressant de constater que le processus de codification, d'uniformisation, de la liturgie à l'époque carolingienne a fortement agi sur l'évolution et le développement de la conscience de la paroisse médiévale ${ }^{102}$. Ce rôle primordial de la liturgie dans la formation de la communauté paroissiale s'est exercé de façon décisive notamment à travers la diffusion des différents livres liturgiques ou de textes propres à tel ou tel rite. Les cérémonies et les formules des actes sacrés s'étant bien fixées par écrit, le clergé, régulier ou séculier, a de mieux en mieux tenu son rôle dans l'encadrement religieux des populations. Ce sont précisément certains aspects de ce rôle que reflète une grande partie des libelli liturgiques.

A partir du IXe siècle, le nombre accru de messes votives, dont les formulaires étaient bien souvent transcrits et diffusés grâce aux libelli, correspond à une orientation nouvelle prise non seulement par la spiritualité monastique, mais aussi par la pratique liturgique dans les paroisses ${ }^{103}$. En milieu monastique, la profusion des messes votives s'explique essentiellement par un usage liturgique où la dévotion personnelle a pris le pas sur la célébration collective

fonctions liturgiques, entre autres. Cette cohabitation active entre moines et prêtres fut parfois à l'origine de graves tensions entre les premiers et l'autorité épiscopale ; voir l'article pionnier de Dom U. BERLIERE, « $L$ ' exercice du ministère paroissial par les moines dans le haut Moyen Age », darns Revue Bénédictine, t. 39, 1927, p. 227 250 , ainsi que différentes études plus récentes, ponctuelles ou générales, parmi lesquelles on retiendra : G. DEVAILLY, « Le clergé régulier et le ministère paroissial », dans Cahiers d'Histoire, t. 20, 1975, p. 259-272 ; J.-F. LEMARIGNIER, "Le monachisme et l'encadrement des campagnes du Royaume de France situées au nord de la Loire, de la fin du $\mathrm{X}^{\mathrm{e}}$ à la fin du $\mathrm{XI}^{\mathrm{e}}$ siècle », dans Le Istituzioni ecclesiastiche della « Societas Christianas 》 dei secoli XI-XII, Milano, 1977 (Miscellanea del Centro di Studi Medioevali, 8), p. 357-405 ; P. ToubERT, «Monachisme et encadrement religieux des campagnes en Italie au $\mathrm{X}^{\mathrm{e}}-\mathrm{XH}$ siècles », ibid., p. 416-441 ; G. ConstABLE, « Monasteries, Rural Churches and the Cura animarum in the Early Middle Ages », dans Cristianizzazione ed organizzazione ecclesiastica delle campagne nell'alto medioevo : espansione e resistenze, Settimane di Studio del Centro Italiano di Studi sull'alto Medioevo, 10-16 avril 1980, Spoleto, 1982, p. 349-389; J.-F. LEMARIGNIER, « Encadrement religieux des campagnes et conjoncture politique dans les régions du Royaume de France situées au nord de la Loire, de Charles le Chauve aux derniers carolingiens (840-987) », ibid., p. 765800 .

102. Voir à ce sujet H. PLATELIE, « La paroisse et son curé jusqu'à la fin du XII" siècle. Orientations de la recherche actuelle », dans L'encadrement religieux des fidèles au Moyen Age et jusqu'au concile de Trente, $109^{\circ}$ Congrès national des Sociétés Savantes, Dijon, 1984, Paris, 1985, t. I, p. 11-26, et l'article très important de A. ANGENENDT, « Die Liturgie und die Organisation des kirchlichen Lebens auf dem Lande , dans Cristianizzazione ed organizzazione, op. cit. en note 101 , p. $169 \mathrm{sq}$. ; surtout p. 170-179 sur les conséquences de la codification de la liturgie et de ses textes sur la formation des paroisses.

103. Voir l'étude fondamentale de A. ANGENENDT, «Missa specialis. Zugleich ein Beitrag zur Entstehung der Privatmessen », dans Frühmittelalterliche Studien, Bd 17, 1982, p. 153-221 ; on pourra aussi consulter avec profit l'ouvrage classique de J.A. JUNGMANN, Missarum Sollemnia, trad. fr., t. I, Paris, 1950, p. 256 sq. 
et ecclésiale. Le moine-prêtre dit sa messe en solitaire, à l'aide d'un libellus missae qui peut prendre des formes modestes, pour son salut ou pour les pécheurs, les vivants, les morts, les pauvres, l'obtention de la pluie, les frères défunts, et bien d'autres thèmes encore $^{104}$. A titre d'exemple, qu'on pourrait multiplier à souhait, citons deux passages des coutumes que l'on observe à Cluny du temps de saint Odilon : "Constitutio monachorum cluniensium de commemoratione omnium fidelium defunctorum : omnes denique sacerdotes ipso die privatim missas caelebraent pro requie omnium animarum fidelium tantumodo cum supradictis collectis pro defunctis » 105 ; ou encore : «Decretum Odilonis abbatis cluniensis de commemoratione fidelium defunctorum : Privatim et publice missas caelebrent pro requie omnium animarum fidelium et duodecim pauperes reficiantur ${ }^{106}$. Bien que les mentions des coutumiers ne précisent pas beaucoup la désignation des livres liturgiques utilisés, il est possible que ces messes fussent célébrées avec des libelli missarum, semblables à ceux repérés dans les catalogues médiévaux et les manuscrits subsistants.

Par cette pratique, les moines-prêtres pouvaient répondre à une demande et des nécessités paroissiales impossibles à remplir par le seul clergé des paroisses qui, comme nous allons le voir, était absorbé par de multiples tâches.

La multiplication des messes votives et des moines-prêtres a permis le développement de la pratique de la messe privée, au caractère principalement pénitentiel ou dévotionnel. De nombreuses études, parfois contradictoires et polémiques, ont montré la place prééminente de cette pratique dans la vie des monastères du haut Moyen Age ${ }^{107}$. Des raisons spirituelles (comme le salut de l'âme) et pénitentielles (pénitence tarifée) amenaient les fidèles à « commander " un certain nombre de messes à un moine-prêtre, qui représentaient aussi un intérêt pécunier pour le monastère. Nous avons vu que des solutions pratiques furent mises au point avec la confection de libelli missarum pour cet usage, tel le ms 610 de l'Arsenal, cas isolé mais certainement représentatif, réalisé à Reichenau à la fin du Xe siècle.

104. Cf. R. GREGOIRE, « La communion des moines-prêtres à la messe d'après les coutumiers monastiques médiévaux », dans Sacris Erudiri, t. 18, 1967/1968, p. 524549.

105. Liber Tramitis Aevi Odilonis abbatis, éd. P. DINTER, $C C M, \mathrm{t} . \mathrm{X}$, Siegburg, 1980 , p. $187, \mathrm{n}^{\circ} 126,1.15-17$.

106. Ibid., p. $199, \mathrm{n}^{\circ} 137,1.15-16$.

107. A. HÄUSSLING, Mönchskonvent und Eucharistiefeier, Münster, 1973 (Liturgiewissenschaftliche Quellen und Forschungen, 58) ; C. VoGEL, « La vie quotidienne des moines-prêtres en Occident à l'époque de la floraison des messes privées ", dans Liturgie, Spiritualité, Cultures, Conférences Saint-Serge, XXIX ${ }^{e}$ Semaine d'études liturgiques, Rome, 1983, p. 341-360, avec bibliographie rétrospective. 
Le prêtre de paroisse avait lui aussi besoin de libelli, peut-être encore plus modestes dans leur aspect matériel que ceux des monastères, dans le cadre d'actions liturgiques primordiales dans la pastorale du haut Moyen Age : l'onction des malades ${ }^{108}$, le viatique, les funérailles ${ }^{109}$, la pénitence ${ }^{110}$. Les libelli offraient le double avantage de ne contenir que ce qui était strictement nécessaire au célébrant dans le cadre d'une action précise, et de pouvoir être facilement emportés avec soi.

Les nombreux capitula episcoporum du haut Moyen Age (IX ${ }^{e_{-}}$ $X^{e}$ siècles), émanant de différents évêques et qui sont des règlements intérieurs destinés à leur diocèse, témoignent de façon particulièrement éclairante et vivante des pratiques pastorales de cette époque ${ }^{111}$. Réglementant parfois sévèrement les pratiques presbytérales, touchant même à la vie privée du prêtre (interdiction de vivre avec une femme, de se réunir trop souvent avec des amis ou de faire des festins, par exemple), les capitula mettent l'accent sur les aspects fondamentaux de la vie religieuse du paroissien : baptême, pénitence, communion, maladie, mort ${ }^{112}$. De très nombreux chapitres de ces capitula entrent dans les détails des conditions du déroulement de ces rites. Bien qu'il ne soit que très rarement fait allusion aux livres dont on se servait pour les célébrations, on peut supposer que bon nombre de libelli ont été utilisés pour l'onction des malades, les funérailles ou encore la pénitence ; les mentions des catalogues médiévaux ainsi que les manuscrits conservés en témoignent. Pour le baptême, on faisait plutôt usage du sacramentaire à l'église, ce qui expliquerait l'absence relative de traces de libelli propres à ce rite, aussi bien dans les catalogues que dans les manuscrits. En revanche, l'onction des malades, le viatique, la pénitence, nécessitaient la pratique

108. Cf. les réflexions de A. TRIACCA, « Le rite de l'impositio manuum super infirmum dans l'ancienne liturgie ambrosienne ", dans La maladie et la mort du chrétien dans la liturgie, Conférences Saint-Serge, XXI ${ }^{e}$ Semaine d'études liturgiques, Rome, 1975, p. 339-360.

109. D. SiCARD, La liturgie de la mort dans l'église latine des origines à la réforme carolingienne, Münster, 1978 (Liturgiewissenschaftliche Quellen und Forschungen, 63) ; ce livre consiste surtout en de nombreuses analyses des formulaires des messes des morts, utilisant bon nombre de libelli, mais le cadre des célébrations est très peu évoqué. Ce manque est compensé par l'article récent de P.-A. FEVRIER, « La mort chrétienne », dans Segni e riti nella chiesa altomedievale occidentale, Settimane di studio del Centro Italiano di Studi sull'alto Medioevo, XXXIII, 11-17 avril 1985, Spoleto, 1987, t. II, p. 881-942.

110. R. KotTJE, « Busspraxis und Bussritus », ibid., t. I, p. 369-395.

111. Sur ce type de document, voir l'introduction de P. BROMMER, "Capitula episcoporum ». Die bischöflichen Kapitularien des 9. und 10. Jahrhunderts, Tumhout, 1985 (Typologie des sources du Moyen Age occidental, fasc. 43).

112. Voir l'étude très stimulante de G. DeVAILLY, « La pastorale en Gaule au IX ${ }^{\mathrm{e}}$ siècle », dans Revue d'histoire de l'Église de France, t. 59, 1973, p. 23-54. 
d'une liturgie itinérante et, par conséquent, de documents appropriés, les libelli, faciles à transporter et peu luxueux.

Dans ces mêmes capitula episcoporum, il est fréquemment question des livres liturgiques que tout prêtre doit posséder. Les livres qui reviennent le plus souvent sont le sacramentaire (liber sacramentorum), le lectionnaire, le psautier, l'antiphonaire (ou le graduel) ; dans la plupart des cas, on indique qu'ills doivent être corrects, probablement du double point de vue du contenu et de leur aspect matériel ${ }^{113}$. Seule une mention dans le capitulaire de Raoul de Bourges fait allusion à des libelli : "Admonendi etiam sunt sacerdotes, ut operam dent, quatenus missalem et lectionarium, psalterium, sive alios libellos sibi necessarios bene correctos habeant... "114. D'autres mentions dans divers capitulaires pourraient faire penser à des libelli pour le baptême, le rite de la sépulture, la consécration des fonts et de l'eau bénite ; mais en l'absence d'indication précise sur les livres, il faut éviter une conclusion trop rapide ${ }^{115}$.

Arrivé au terme de notre enquête, nous espérons avoir montré tout l'intérêt des libelli pour une vision plus large des pratiques liturgiques du haut Moyen Age.

Dans le domaine de l'histoire de la liturgie, les libelli apparaissent comme un type de document dont l'importance est capitale pour la genèse des livres liturgiques. Leur histoire et leur

113. Voir par exemple les mentions des capitula suivants : Hildegard de Meaux, vers 868, Capitula Episcoporum, I, M.G.H., Cap. Ep., I, éd. P. BROMMER, Hannover, 1984 (cité désormais Cap. Ep. I), p. 198, ch. 3 ; Gautier d'Orléans, vers 869/870, Cap. Ep. I, p. 189-193 ; Hatton de Bâle, entre 806 et 823, Cap. Ep. I, p. 211 ; Riculf de Soissons, après $889, P L ., 131$, col. 17 ; et des textes non attribués comme ceux du début du $\mathrm{IX}^{\mathrm{e}}$ siècle, étudiés par $\mathrm{R}$. AMIET, « Une Admonitio synodalis carolingienne », dans Medieval Studies, t. 26, 1964, p. 12-82, spéc. p. 46, $\mathrm{n}^{\circ} 25$ et par W. HartmanN, entre 829 et 831 , « Neue Texte zur bischöflichen Reformgesetzgebung aus den Jahren $829 / 831$ ", dans Deutsches Archiv für Erforschung des Mittelalters, Jhrg. 35, 1979, p. 368-394, spéc. p. 385. Sur ces différents capitula, on pourra aussi relire les pages de C. DE CLERCQ, La législation religieuse franque de Louis le Pieux à la fin du $X^{e}$ siècle, t. I, Anvers, 1958, p. 339 sq.

114. Capitulaire daté entre 853 et 866 , Cap. Ep. I, p. 237. P. Brommer a bien montré que ce capitulaire s'inspirait largement des capitulaires de Théodulf d'Orléans, notamment, mais il n'en est rien pour ce passage, cf. P. BROMMER, « Die Quellen der Capitula Radulfs von Bourges », dans Francia, Bd 5, 1977, p. 27-43.

115. Comme celle du capitulaire de Riculf de Soissons, après 889 : "Consignationem vero infantum, tam masculorum quam feminarum, unius vel plurium, et consecrationem fontis, et aquae per domo spargendae, et animae commendationem, et orationes ad mortuum sepeliendum habeatis descripta distincte et veraciter, et frequenti usu ea meditemini, ut correcte et irreprehensibiliter ea tam super masculum quam super feminam proferre sciatis. Supradictam vero consignationem infantum volumus ut quadrifarie scribendo divitatis, super singularem scilicet masculum et singularem feminam, itemque super masculos plures et plures feminas, sicut nos vobis, christo propitio, descripta trademus », P.L., 131, col. 17. 
typologie éclairent d'une lumière nouvelle l'évolution et la formation des livres du culte chrétien à une époque de mise en place et de codification de la liturgie occidentale, l'époque carolingienne et ses prolongements. On a vu également de quelle façon l'ecclésiologie de la liturgie du haut Moyen Age a, par certains aspects, été déterminée par les livres que l'on utilisait dans l'exercice du culte. Dans ce domaine, les libelli ont là encore joué un rôle de première importance, notamment par la possibilité qu'ils offraient de multiplier les petits livrets pour les différents rites et les acteurs de la liturgie.

Nous espérons avoir également montré tout l'intérêt de ces livrets pour l'histoire de l'Eglise du haut Moyen Age. Rappelons simplement deux exemples. D'une part, la multiplication des messes privées et des moines-prêtres, phénomène capital dans l'évolution de la place du moine et des monastères dans l'Occident médiéval, qui a engendré, sur le plan pratique, la conception d'un nouveau type de manuscrit liturgique, de libelli, spécialement conçu pour la célébration privée et dévotionnelle du moine.

Le second exemple est l'encadrement religieux des campagnes et la formation de la notion de paroisse au cours de l'époque carolingienne et au Xe siècle. Certains libelli ou mentions de libelli que nous avons présentés, témoignent concrètement de l'existence, à cette époque, d'une liturgie bien spécifique au prêtre, dans le cadre bien limité de sa paroisse. Il s'agit là, sans aucun doute, de témoins qui viennent enrichir notre connaissance - bien lacunaire, il faut l'avouer, parce que basée sur une documentation fragmentaire - de la vie des paroisses du haut Moyen Age.

Éric PALAZZZO

Institut supérieur de Liturgie de Paris Mission historique française en Allemagne 